\title{
Large compact clusters and fast dynamics in coupled nonequilibrium systems
}

\author{
Shauri Chakraborty ${ }^{1}$, Sukla Pal ${ }^{1}$, Sakuntala Chatterjee ${ }^{1}$ and Mustansir Barma ${ }^{2}$ \\ 1 Department of Theoretical Sciences, S.N. Bose National Centre for Basic Sciences, \\ Block - JD, Sector - III, Salt Lake, Kolkata 700098, India \\ 2 TIFR Centre for Interdisciplinary Sciences, 21 Brundavan Colony, \\ Osman Sagar Road, Narsingi, Hyderabad 500075, India.
}

\begin{abstract}
We demonstrate particle clustering on macroscopic scales in a coupled nonequilibrium system where two species of particles are advected by a fluctuating landscape and modify the landscape in the process. The phase diagram generated by varying the particle-landscape coupling, valid for all particle density and in both one and two dimensions, shows novel nonequilibrium phases. While particle species are completely phase separated, the landscape develops macroscopically ordered regions coexisting with a disordered region, resulting in coarsening and steady state dynamics on time scales which grow algebraically with size, not seen earlier in systems with pure domains.
\end{abstract}

PACS numbers: 05.40.-a, 64.75.Gh, 68.43.Jk

Particle clustering is important in many natural physical and biological phenomena, for instance, the formation of sediments [1] and protein clustering on a biological membrane 2]. Evidently, it is important to understand processes that cause clustering in different physical contexts, and how these processes influence the properties of the cluster and the time taken to form it. Often, large-scale clustering is driven by interactions with an external medium which itself has correlations in space and time [3 [5]. An important physical effect in such systems is the back-influence of the particles on the medium. This interaction can aid clustering, or destroy it. If a cluster does form, it may be compact and robust, or a dynamic object that undergoes constant reorganization. The formation time may grow exponentially with the size, or as a power law. Given this wealth of possibilities, it is important to look for an understanding, within simple models, of the circumstances under which different sorts of macroscopically clustered states occur.

In this letter, we derive the phase diagram of a simple model system as we vary the interaction between the environment and particles. In the process, we unmask a novel non-equilibrium phase of particles with compact clustering and rich and rapid dynamics coexisting with a macroscopically organized landscape. The model has partial overlap with the lattice gas model of Lahiri and Ramaswamy (LR) for sedimenting colloidal crystals [6, 7], but the new phases manifest themselves outside the LR regime. Our results hold in both one and two dimensions.

The model consists of two sets of particles moving stochastically in a fluctuating potential energy landscape. Particles try to minimize their energy by (a) moving along the local potential gradient of the landscape and (b) modifying the landscape around them in such a way as to lower the energy further. The model is generic but we discuss it in the language of particles confined to move on a fluctuating surface in the presence of gravity, where the particles can locally distort the surface shape to further lower the energy (see Fig. 11). One of the particle species is considered lighter and the other is heavier; we use the name LH (Light-heavy) model to describe the system. Process (b) affects the landscape dynamics quite differently in parts which are rich or poor in one species of particle, ultimately resulting in the formation of distinct macroscopic regions, each corresponding to a phase. Our study reveals a rich set of phenomena: strong phase separation with fluctuationless phases for particles, but a different sort of organization for the landscape; a rapid approach to the steady state; and intricate steady state dynamics of the interfaces between phases, with three distinct temporal regimes.

There has been a recent surge of activity in the field of coupled driven diffusive systems [8 -10], and it is useful to view our work in this context. This activity has resulted in a catalogue of universality classes which describe how propagating modes in these systems decay in time. The modes themselves are defined by diagonalizing coupled hydrodynamic equations to linear order, with eigenvalues giving their speeds of propagation. The disordered phase of our system is indeed described by this theory. But the ordered phases of primary interest to us correspond to complex eigenvalues at the linear level [11]; the imaginary parts signal instabilities, heralding macroscopic phase separation. However, such linear analysis cannot reveal the characteristics of the final phases, which can and do differ from each other in fundamental respects. Our results provide the necessary characterization and thus contribute to the important goal of classifying ordered phases in coupled driven diffusive systems.

In a nutshell, the quintessentially nonequilibrium states found here exhibit phase separation, with qualitatively different types of ordering for the particles and landscape, quite unlike systems known earlier. In particular, particles display strong phase separation [7] characterized by pure, fluctuationless phases, which cohabit with three macroscopic regions of the surface, two of which possess long range order, while the third does not. These findings differ markedly from the strongly phase separated states found earlier in the LR and ABC models [7, 12] and imply strong changes for both static and dynamical properties. Notably, the approach to our steady state is rapid, with a coarsening time that grows as a power law of size, as opposed to the much slower exponentially growing time scales found earlier. 


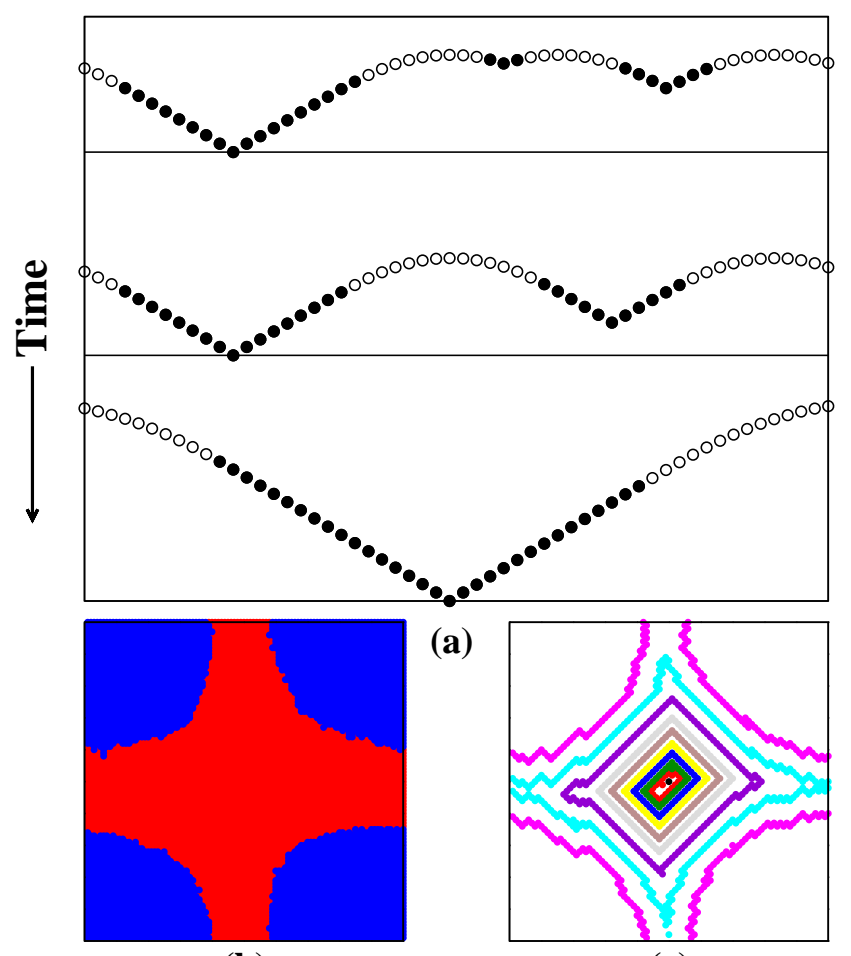

(b)

(c)

FIG. 1: Schematic presentation of different phases in one and two dimensions. (a) shows the coarsening mechanism in one dimension for $b^{\prime} \leq 0$. $H(L)$ particles are shown by solid (hollow) circles. (b) and (c) show typical configurations in two dimensions. In (b) $H(L)$ cluster shown by red (blue) color and (c) shows the equal height contours.

The LH model consists of two coupled driven diffusive systems, with conserved quantities. This is a lattice model of $H$ (heavier) and $L$ (lighter) particles with damped motion under gravity and residing on a fluctuating surface. The local dynamics of the particles and the surface are coupled: $H$ and $L$ particles at neighboring sites may interchange locations, and do so preferentially if the local tilt of the surface favors a downward move for $H$. Particles reside on lattice sites and interact via hard-core exclusion: a site holds at most one particle $(H$ or $L)$. If the symbols / and $\backslash$ indicate upward and downward tilts of the surface, respectively, then the particles follow the dynamics:

$$
\begin{aligned}
& W(H \backslash L \rightarrow L \backslash H)=D+a \\
& W(L \backslash H \rightarrow H \backslash L)=D-a \\
& W(H / L \rightarrow L / H)=D-a \\
& W(L / H \rightarrow H / L)=D+a
\end{aligned}
$$

where $W$ denotes the probability per unit time for a particular process to occur. This dynamics conserves the total number of $H$ (and $L$ ) particles. Under the weight of the particles, a local hill on the surface gets pushed downward, at a higher rate by $H$ than by $L$. In one dimension, the surface consists of a chain with $N$ sites. The lattice bonds representing discrete surface elements, can have two possible orientations with slopes $\tau_{i+1 / 2}= \pm 1$, which are called upslope and downslope bonds, respectively. In one dimension surface dynamics can be represented as

$$
\begin{aligned}
W(/ H \backslash \rightarrow \backslash H /) & =E+b \\
W(\backslash H / \rightarrow / H \backslash) & =E-b \\
W(/ L \backslash \rightarrow \backslash L /) & =E-b^{\prime} \\
W(\backslash L / \rightarrow / L \backslash) & =E+b^{\prime}
\end{aligned}
$$

This dynamics conserves the overall slope. In two dimensions the surface consists of a square lattice and height of a site can change, provided all four neighboring sites are at the same height [13, 14]. We consider periodic boundary conditions with no overall tilt of the surface.

Figure 2 shows the phase diagram of the system in $b b^{\prime}$ plane, with $a$ taken to be positive. It follows from Eq. 2 that interchanging $b$ and $b^{\prime}$ is tantamount to interchanging the $H$ and $L$ species. Hence we consider positive $b$, while $b^{\prime}$ can be positive or negative or zero. 


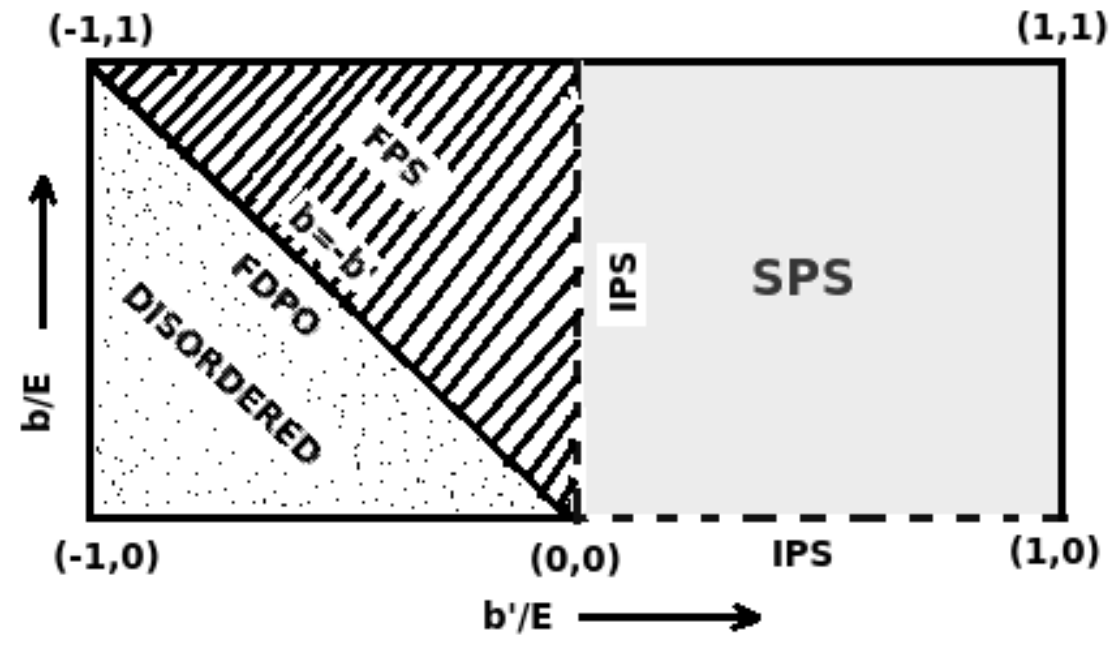

FIG. 2: Phase diagram in the $b-b^{\prime}$ plane. For $b>0$ and $b^{\prime}>0$, the system shows SPS. The dotted horizontal and vertical lines are related to each other via interchange of the two particle species. On these lines the system is in IPS phase. The striped region $\left(-b<b^{\prime}<0\right)$ represent FPS phase. $b=-b^{\prime}$ line corresponds to FDPO phase. The dotted region $\left(b^{\prime}<-b\right)$ correspond to disordered phase.

Strong Phase Separation (SPS): The right half of the phase diagram, $b^{\prime}>0$, corresponds to the LR model [ [6, 7], as appropriate to sedimenting colloidal crystals [6]. In this regime, the light particles tend to move the surface upward. In steady state, the upslope and downslope surface bonds phase separate to form a single macroscopic valley and hill, which hold all the $H$ and $L$ particles, respectively, in separated clusters. Both particles and tilts show SPS; the approach to steady state is extremely slow (involving times $T_{\text {relax }} \sim \exp (\alpha N)$ ) owing to the formation of large metastable barriers.

In the left half of the phase diagram in Fig. 2 we have $b^{\prime}<0$, which means that the part of the surface occupied by $L$ particles shows a downward drift. As discussed below, different phases are obtained depending on whether this velocity is larger than, smaller than, or the same as the velocity imparted by the $H$ particles.

Disordered Phase: When the $L$ particles push the surface faster (dotted region in Fig. 2), neither of the particle species nor the tilts shows long-range order. Interesting dynamical aspects of this wave-carrying disordered phase will be presented elsewhere 15.

Fluctuation-dominated Phase Ordering (FDPO): On the line $b=-b^{\prime}$, both $L$ and $H$ particles push the landscape down at the same rate. This implies Kardar-Parisi-Zhang dynamics for the surface while $L-H$ exchange rules (Eq. 11) imply that $H$-particles tend to collect in local valleys. This reduces to the passive scalar problem studied earlier, in which particles exhibit FDPO, characterized by singularities of the 2-point correlations and giant fluctuations of the density [16, 17]. Interestingly, this phase boundary can be identified exactly by looking for the onset of complex eigenvalues in a linear stability analysis of the coupled hydrodynamic equations for the landscape and the particles [14].

Fast-fall with Phase Separation (FPS): When $L$ particles push the surface down at a slower rate than H's, a new phase ensues (shown striped in Fig. 21). In steady state, $L$ and $H$ particles separate completely as in SPS. The surface underlying the $H$-cluster forms a macroscopic valley but unlike SPS, the phases are not pure, e.g. the macroscopic majority-upslope region accommodates a finite fraction $(1-m)$ of downslope bonds. The majority-upslope region in turn acts as a 'tilt reservoir' which drives a finite tilt current through the part of the surface which holds $L$ particles. By identifying an upslope (downslope) bond with a particle (hole) we identify the phase as the maximal current phase in an open-chain asymmetric exclusion process [14, 18]. Consequently, near the edges of the $L$-domain the tilt density $\rho$ shows an algebraic $1 / \sqrt{r}$ variation, while in the bulk $\rho \approx 1 / 2$ [14]. Equating the tilt current $J=2 b m(1-m)$ in the two arms holding the $H$ particles with that in the maximal current phase $J^{\prime}=2 b^{\prime} / 4$ in the $L$-rich portion, we deduce $4 m(1-m)=b^{\prime} / b$, a relation we have verified numerically. The presence of a finite tilt current through the system results in a finite downward velocity of the surface and in steady state, the full surface moves downward at finite speed, preserving the macroscopic valley and disordered tilt region, along with the pure domains of $H$ and $L$ particles (Fig. 17).

Infinitesimal-fall with Phase Separation (IPS): For $b^{\prime}=0$ (the vertical dashed line in Fig. 22) the local fluctuations in the surface occupied by $L$ particles are of the symmetric Edwards-Wilkinson type. In this phase, the $H$ and $L$ particles again form pure domains. The surface beneath the $H$-cluster has the shape of a deep valley consisting of 


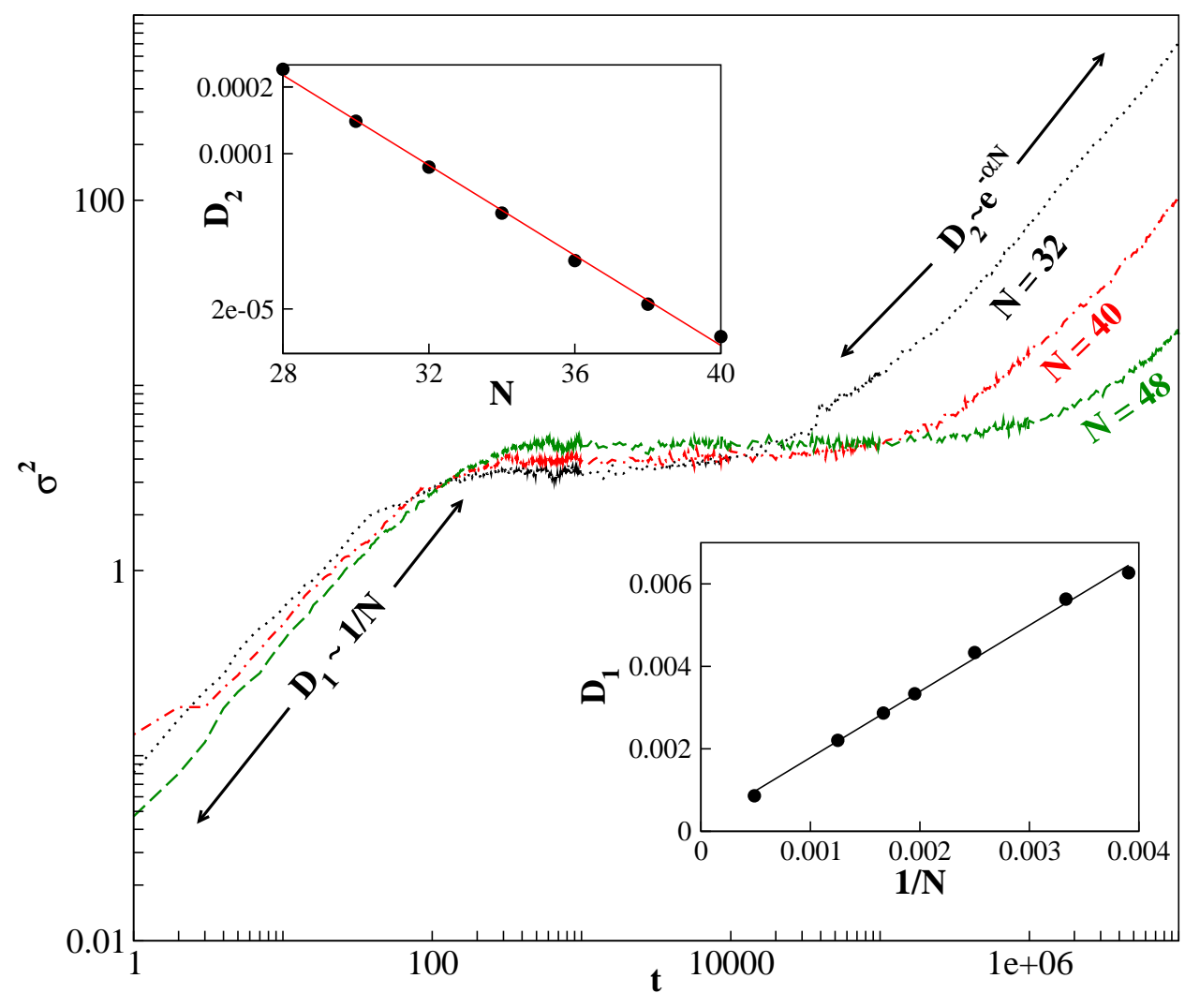

FIG. 3: Occurrence of three regimes in the steady state dynamics of the deepest point of the valley. Main plot: Mean squared displacement of the deepest point of the valley as a function of time. The displacement shows an initial diffusive growth, followed by a plateau, and finally another diffusive regime at large time. Bottom Inset: Small time diffusivity $D_{1} \sim 1 / N$. Top Inset: For large time, the diffusivity $D_{2} \sim e^{-\alpha N}$, with $\alpha \simeq 0.26$. These data correspond to $b^{\prime}=0, b=E, a=D$ and have been averaged over 5000 steady state configurations.

pure domains of upslope and downslope bonds. By contrast, the surface occupied by the $L$-cluster in this case behaves like an open-chain symmetric exclusion process connected to the two reservoirs of upslope and downslope tilts at the two ends. Thus the tilt density varies linearly in this region with a gradient $\sim 1 / N[14$, 19], leading to a tilt current and an infinitesimal downward velocity $\sim 1 / N$ of the entire landscape. We schematically show a typical configuration in Fig. 1h.

The general properties of the phases discussed above remain valid even in two dimensions, where $H$ and $L$ particles form compact clusters. The shape of these two dimensional clusters depends on the topography of the surface heights. As in one dimension, we find a deep valley that holds the $H$-cluster. Measured from the bottom-most point, the height increases linearly in both $x$ and $y$ directions and it is easy to see that in this case the equal height contours have the shape of a diamond. In Fig. 10 and 1k, we show some representative configurations.

The way in which the landscape is organized in the IPS and FPS phases has a profound influence on dynamical properties. For instance, although the centre of mass of the $H$ cluster remains stationary for a long time, the landscape immediately below it undulates in time, leading to three distinct temporal regimes in steady state. These are captured by monitoring the mean-squared displacement $\sigma^{2}$ of the deepest point of the valley. Figure 3 presents the data for $b^{\prime}=0$. At small time $t \ll N^{2}$, we find $\sigma^{2}$ grows diffusively with a diffusion constant $D_{1} \sim 1 / N$. But after times of order $N^{2}$, a plateau for $\sigma^{2}$ is reached at a value $\sim N$. From a simple consideration of the total (gravitational) energy of the $H$ particles, it is easy to show that when the deepest point coincides with the centre of mass of the $H$ cluster, the energy is minimum. Any displacement from this position gives rise to a restoring force that scales linearly with the displacement. The motion of the deepest point is thus described by an Ornstein-Uhlenbeck process [20]; consequently, the deepest point diffuses within a region of size $\sqrt{N}$ around the $H$ cluster centre of mass [14]. Finally, at very large $t$, the $H$ cluster itself moves diffusively around the system and the valley moves along with it (see [14]). The mean-squared displacement of the deepest point in this regime has a diffusion coefficient $D_{2} \sim e^{-\alpha N}$.

Another important aspect of the dynamics concerns the relaxation to steady state starting from an initially disordered state. Interestingly, IPS and FPS phases show an enormous reduction in this relaxation time, as compared to 

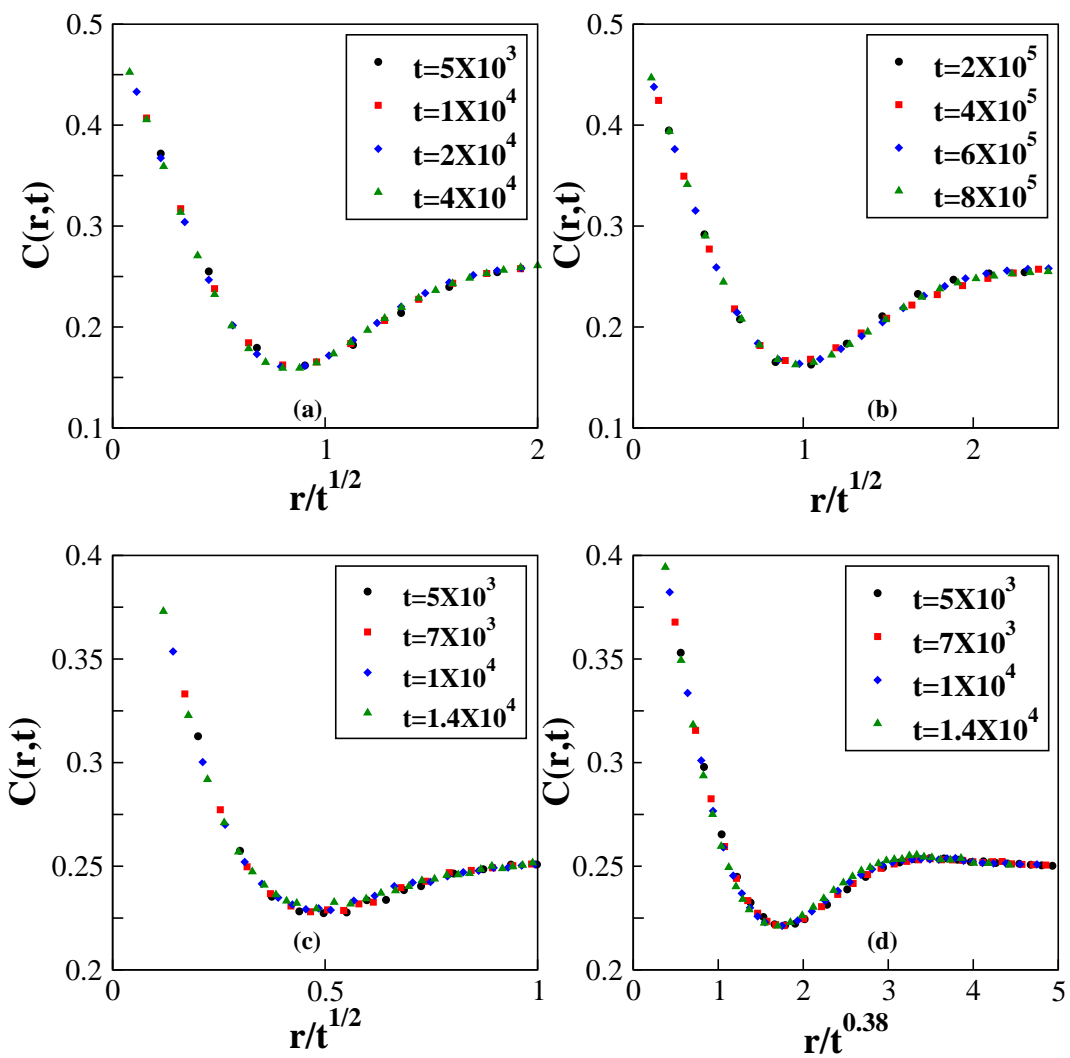

FIG. 4: Scaling of particle density correlation in the coarsening phase. in both one and two dimensions. The equal time density correlation for the particles $C(r, t)$ shows a collapse when $r$ is scaled by $\mathcal{L}(t) \sim t^{1 / z}$. (a) and (c) show scaled data for $b=0.5, b^{\prime}=0$ in one and two dimensions, respectively. Here we find $z \simeq 2$. (b) and (d) show similar plots for $b=0.3, b^{\prime}=-0.2$. Here, in one dimension, $z \simeq 2$ and in two dimensions $z \simeq 2.6$. We have used $N=1024(\mathrm{a}), N=16384(\mathrm{~b})$ and $N=256 \times 256((\mathrm{c})$ and $(d))$ here.

earlier known examples of SPS as in the LR and ABC models [7, 12]. For $b^{\prime}>0$ (LR model), the landscape occupied by an $L$-cluster tends to move upwards and forms a hill, while an $H$-cluster pushes the landscape down and forms a valley. For two adjacent valleys with $H$-clusters to merge, the time to dissolve the intermediate hill containing the $L$-cluster grows exponentially with the size of the $L$-cluster, and hence the final SPS state is reached over a time-scale $e^{\gamma N}$. By contrast, in the FPS and IPS $\left(b^{\prime} \leq 0\right)$ phases, the landscape is organized differently, and this leads to fast relaxation, with times growing as $N^{z}$. This is because the part of the landscape beneath the intervening $L$ cluster either shows symmetric fluctuations (for $b^{\prime}=0$ ) or gets pushed downward (for $b^{\prime}<0$ ). Figure 4 shows the scaling collapse of the equal time density correlation function for $H$ particles when separations are scaled by the coarsening length scale $\mathcal{L}(t)$, which is found to grow as $t^{1 / z}$. In IPS phase $\left(b^{\prime}=0\right)$ we find $z=2$ in both one and two dimensions. The FPS phase $\left(-b<b^{\prime}<0\right)$ shows $z \simeq 2$ in one dimension for very large $N$ and $t$ while for smaller values of these variables, our data show finite size effects (see 14] for details). In two dimensions for FPS, we measured $z \simeq 2.6$, for the largest values of $N$ and $t$ we could access. Our plots in Fig. 4 constitute the first observation of algebraic coarsening for completely phase separated systems, and stand in strong contrast to the ultraslow logarithmic coarsening observed in the LR and ABC models 7, 12]. Underlying the speed-up of the coarsening process is a simple mechanism, namely the formation of disordered segments of the landscape between ordered clusters. These segments generate fluctuations which allow mergers of ordered regions to occur on a rapid time scale.

To summarize, we have considered coupled driven systems consisting of two species of particles being advected by an energy landscape whose dynamics is in turn influenced by the particles. The differential interaction between the landscape and the two particle species gives rise to different phases in the system as the interaction parameters are varied. We have demonstrated the occurrence of new phases with fast dynamics, where both the particles and the landscape show long range order and the composite system is in a nonequilibrium current-carrying state.

We conclude with a discussion of the implications of our work for modeling in biophysical contexts. It is known that on the cell membrane, various proteins and lipids like integrins, T-cell receptors are present in the form of nanoclusters [21, 22]. This clustering is shown to be induced by the cortical actin cytoskeleton [2, 23], and within a recent 
theoretical model of the process, an FDPO state has been observed [24]. There is now experimental evidence that the actin cytoskeleton also gets reorganized by these membrane components 22, 25]. This raises the interesting possibility of new phases arising if the treatment of [24] is extended to account for the back action of membrane components on the cytoskeleton.

We acknowledge useful discussions with T. Sadhu, M. Rao, and A. Das. The computational facility used in this work was provided through Thematic Unit of Excellence on Computational Materials Science, funded by Nanomission, Department of Science and Technology, India.

[1] S. Ramaswamy,Advances in Physics 50, 297 (2001)

[2] K. Gowrishankar, S. Ghosh, S. Saha, C. Rumamol, S. Mayor, and M. Rao, Cell 149, 1353 (2012).

[3] J. M. Deutsch, J. Phys. A 18, 1449 (1985)

[4] M. Wilkinson and B. Mehlig, Phys. Rev. E 68, 040101 (2003)

[5] B. Drossel and M. Kardar, Phys. Rev. Lett. 85, 614 (2000); A. Nagar, M. Barma and S. N. Majumdar, Phys. Rev. Lett. 94, $240601(2005)$

[6] R. Lahiri, S. Ramaswamy, Phys. Rev. Lett. 79, 1150 (1997).

[7] R. Lahiri, M. Barma and S. Ramaswamy, Phys. Rev. E 61, 1648 (2000).

[8] H. van Beijeren, Phys. Rev. Lett. 108, 180601 (2012).

[9] P. Ferrari, S. Sasamoto and H. Spohn, J. Stat. Phys. 153377 (2013).

[10] V. Popkov, A. Schadschneider, J. Schmidt and G. Schütz, Proc. Natl. Acad. Sci. USA, 112, 12645 (2015)

[11] S. Ramaswamy, M. Barma, D. Das and A. Basu, Phase Transit. 75, 363 (2002)

[12] M.R. Evans, Y. Kafri, H.M. Koduvely, and D. Mukamel, Phys. Rev. Lett. 80, 425 (1998).

[13] G. Manoj and M. Barma, J. Stat. Phys. 1101305 (2013).

[14] See supplemental material for details of calculations and additional data from simulations.

[15] S. Chakraborty, S. Chatterjee and M. Barma in preparation

[16] D. Das and M. Barma, Phys. Rev. Lett. 85, 1602 (2000); D Das, M Barma and S. N. Majumdar, Phys. Rev. E 64, 046126 (2001); S. Chatterjee and M. Barma, Phys. Rev. E 73, 011107 (2006).

[17] R. Kapri, M. Bandopadhyay and M. Barma, Phys. Rev. E 93, 012117 (2016).

[18] B. Derrida, E. Domany and D. Mukamel, J. Stat. Phys, 69667 (1992).

[19] B. Derrida, J.L. Lebowitz, and E.R. Speer, J. Stat. Phys. 107, 599 (2002).

[20] G.E. Uhlenbeck and L.S. Ornstein Phys. Rev. 36, 823 (1930).

[21] G. J. Bakker, C. Eich, J. A. Torreno-Pina, R. Diez-Ahedo, G. Perez-Samper, T. S. van Zanten, C. G. Figdor, A. Cambi and M. F. Garcia-Parajo, Proc. Natl. Acad. Sci. USA, 109, 4869 (2012).

[22] C. Yu, J. B. K. Law, M. Suryana, H. Y. Low and M. P. Sheetz, Proc. Natl. Acad. Sci. USA, 108, 20585 (2011).

[23] D. Goswami, K. Gowrishankar, S. Bilgrami, S. Ghosh, R. Raghupathy, R. Chadda, R. Vishwakarma, M. Rao, and S. Mayor, Cell 135, 1085 (2008).

[24] A. Das, A. Polley and Madan Rao, Phys. Rev. Lett. 116, 068306 (2016).

[25] R. Varma, G. Campi, T. Yokosuka, T. Saito and M. L. Dustin, Immunity 25, 117 (2006).

\section{Appendix A: Simulation details in two dimensions and finite size effects}

The surface in two dimensions is simulated through a discrete solid-on-solid algorithm, where the height difference between the nearest neigbours on a square lattice is maintained at \pm 1 . Let $h(i, j)$ denote the height of the site $(i, j)$ on the lattice. This site is said to be on a local hill, if all its four neigbours with coordinates $(i \pm 1, j)$ and $(i, j \pm 1)$ have height $h(i, j)-1$. Similarly, the site $(i, j)$ is said to be in a valley when the neighbors have height $h(i, j)+1$. The two dimensional surface evolves in time by switching between the hills and the valleys. In our model, a site is selected at random. If it is on a hill, then it can flip to a valley when its height gets reduced by two units. This flipping rate is $(E+b)$ when the site is occupied by an $H$ particle and $\left(E-b^{\prime}\right)$ when it is occupied by an $L$ particle. Similarly, if the chosen site happens to be in a valley, then its height can increase by two units and it becomes a hill. The switching rate in this case is $(E-b)$ when an $H$ particle is present, and $\left(E+b^{\prime}\right)$ when an $L$ particle is present. For updating the particles, we select a bond (horizontal or vertical) of the square lattice. If the two sites adjacent to the bond are occupied by two different species of particles, then their positions are exchanged with rate $(D+a)$ if after the exchange, height of the $H$ particle decreases. The reverse exchange occurs with rate $(D-a)$.

In our simulation, we start with a flat initial configuration of the surface and random configuration for the particles and evolve the system in time, following the algorithm described above. In steady state the surface develops a single valley where the equal height contours resemble diamonds, shown in Fig. 1c in the main paper. However, in certain cases, we also observe another type of state, where a completely different height topography emerges. Instead of a single minimum seen for diamond shaped contours, we find a line of minima and height increases along the direction 
perpendicular to this line. In other words, the surface has the shape of a trench. The equal height contours and the perimeter of the $H$-cluster for such a configuration is shown in Fig. A-1. We have verified that such wedge-like configurations result from finite size effects in our system with periodic boundary conditions and for larger systems only diamond-shaped contours are found. 
(A)

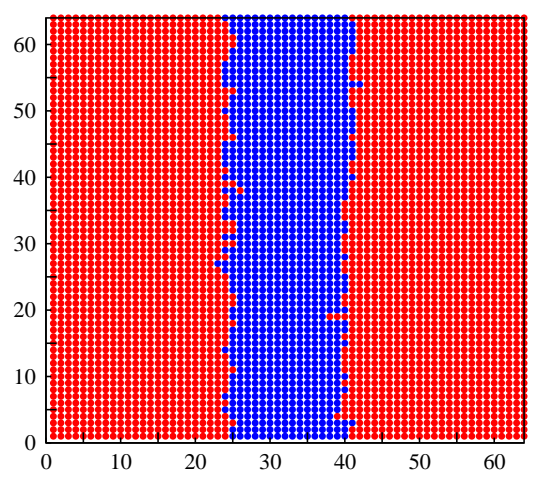

(B)

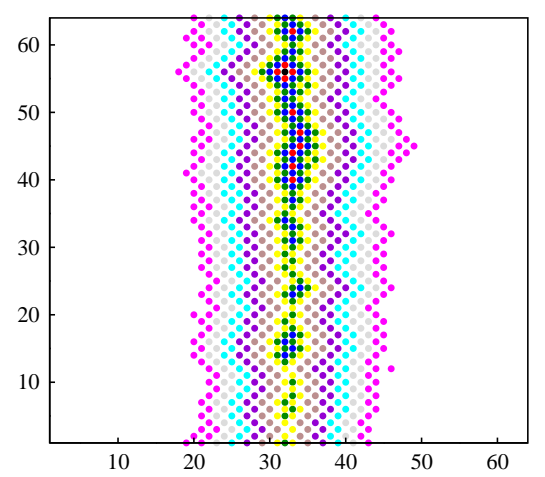

FIG. A-1: Representative plots for wedge type configurations in the IPS phase in two dimensions on a $64 \times 64$ square lattice. In (A) $H(L)$ clusters are shown by red(blue) color, while (B) shows equal height contour plots.

\section{Appendix B: Linear stability analysis of the coupled hydrodynamic equations}

From the dynamical rules shown in Eqs. 1 and 2 in main text, the particle current and the tilt current can be written as

$$
\begin{aligned}
J_{\rho} & =2 a \rho(x, t)(1-\rho(x, t))(1-2 m(x, t)) \\
J_{m} & =m(x, t)(1-m(x, t))\left[2 \rho(x, t)\left(b+b^{\prime}\right)-2 b^{\prime}\right]
\end{aligned}
$$

$\rho(x, t)$ and $m(x, t)$ denote the coarse-grained densities of the $H$ particles and the upslope bonds respectively. We write each density as sum of the average value and a small perturbation: $\rho(x, t)=\rho_{0}+\delta \rho(x, t)$ and $m(x, t)=m_{0}+\delta m(x, t)$ and expand currents in Eq. B-1 upto linear order in $\delta \rho$ and $\delta m$. This gives us the continuity equation

$$
\frac{\partial \vec{u}}{\partial t}+A \frac{\partial \vec{u}}{\partial x}=0
$$

where we have used the compact notation $\vec{u}$, denoting a column vector with components $\rho$ and $m$. The matrix $A$ is defined as

$$
A=\left[\begin{array}{ll}
\frac{\partial J_{\rho}}{\partial \rho} & \frac{\partial J_{\rho}}{\partial m} \\
\frac{\partial J_{m}}{\partial \rho} & \frac{\partial J_{m}}{\partial m}
\end{array}\right]=\left[\begin{array}{lc}
2 a\left(1-2 \rho_{0}\right)\left(1-2 m_{0}\right) & -4 a \rho_{0}\left(1-\rho_{0}\right) \\
2 m_{0}\left(1-m_{0}\right)\left(b+b^{\prime}\right) & 2 \rho_{0}\left(1-2 m_{0}\right)\left(b+b^{\prime}\right)-2 b^{\prime}\left(1-2 m_{0}\right)
\end{array}\right]
$$

For an untilted surface, $m_{0}=1 / 2$, and the eigenvalues are

$$
\lambda= \pm \sqrt{-2 a \rho_{0}\left(1-\rho_{0}\right)\left(b+b^{\prime}\right)}
$$

For $b>-b^{\prime}$, one has imaginary eigenvalues, indicating linear instability. In this case, the system shows macroscopically ordered phases. For $b<-b^{\prime}$, the eigenvalues are real and the system is disordered. The $b=-b^{\prime}$ straight line separates the ordered and the disordered phase and on this line, the system exhibit FDPO.

\section{Appendix C: Static Correlations in Steady State}

In Figs C-1 and C-2 we present our results on the static correlation functions for the particles and for the surface bonds in one dimension. Figure C-1 corresponds to IPS phase and Fig. C-2 correspond to FPS phase. Here we have considered equal number of $H$ and $L$ particles and all distances have been measured from the centre of mass of the $H$-cluster. $\rho(r, N)$ and $S^{+}(r, N)$ denote, respectively, the density of $H$ particles and the probability to find an upslope bond at a distance $r$ from the centre of mass. 

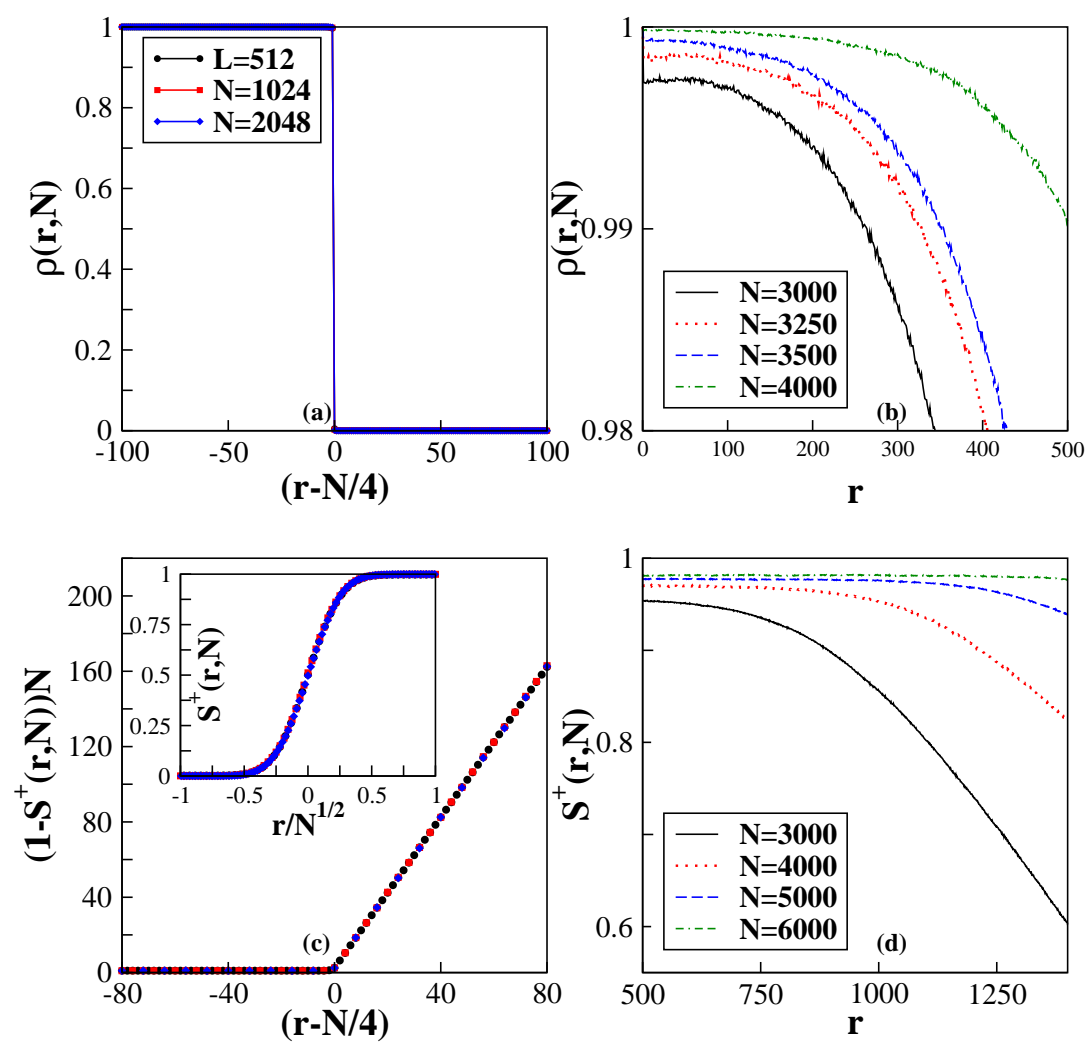

FIG. C-1: Static correlation functions in IPS phase. (a): Density of $H$ particles $\rho(r, N)$ at $q=0$. The density changes sharply from 1 to 0 indicating pure phases of $H$ and $L$ particles. (b): For $q=(D-a) /(D+a)=0.99$ ( see Eq. 1 in the main text), $H$ particle density approaches 1 as $N$ is increased. This confirms the existence of a pure phase in the thermodynamic limit for all $q<1$. (c) Main plot: For $r \gg \sqrt{N}$ a scaling collapse is obtained for different system sizes when $\left[1-S^{+}(r, N)\right] N$ is plotted against $(r-N / 4)$. Inset shows that as $r$ changes sign, $S^{+}(r, N)$ shows a transition from 0 to 1 across the domain boundary, which is at the deepest point of the valley. Due to diffusive motion of the domain boundary, $S^{+}(r, N)$ is a scaling function of $r / \sqrt{N}$ in this region. In main plot and inset we show data for $N=512$ (black circles), 1024 (red squares) and 2048 (blue diamonds). (d): For $q=0.99$ and $0 \ll r \ll N / 4 S^{+}(r, N) \rightarrow 1$ as $N$ is increased. These data have been averaged over at least $3 \times 10^{5}$ steady state configurations. 

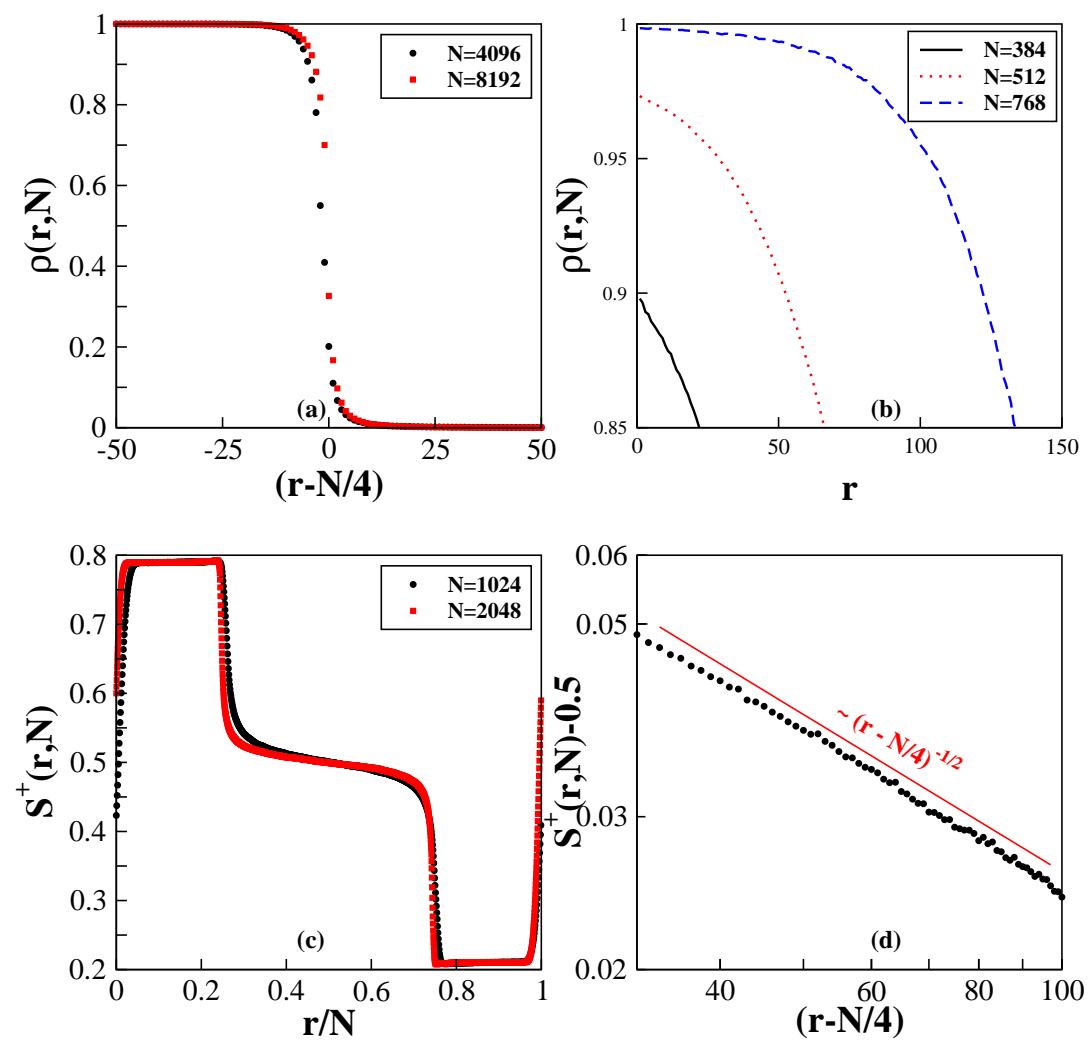

FIG. C-2: Static correlation functions in FPS phase with $b=0.3$ and $b^{\prime}=-0.2$. (a): Density of $H$ particles $\rho(r, N)$. The density changes sharply from 1 to 0 indicating pure phases of $H$ and $L$ particles. (b): For $q=(D-a) /(D+a)=0.92$ (see Eq. 1 in main text), $\rho(r, N)$ approaches 1 as $N$ is increased. This demonstrates existence of pure $L$ and $H$ domains in the thermodynamic limit, as long as $q<1$, i.e. the $H$ particles preferentially slide downward. (c): $S^{+}(r, N)$ for small $r$ remains constant at a value which is less than unity. This indicates that the upslope and downslope bonds underlying $H$-cluster undergo phase separation but no pure phases are formed. The variation of $S^{+}(r, N)$ in the region occupied by $L$ cluster shows an algebraic decay to a disordered phase, as expected for maximal current phase of an open-chain ASEP. (d): The algebraic decay of $S^{+}(r, N)$ occurs with an exponent $1 / 2$, consistent with earlier known results for maximal current phase. These data have been averaged over at least $10^{6}$ steady state configurations.

\section{Appendix D: Mechanisms responsible for two distinct time scales in valley dynamics}

In this section we explain the mechanisms for the short and large time valley dynamics. The deepest point of the valley lies at the boundary between two pure domains of upslope and downslope surface bonds and these pure domains extend upto the edges of the $H$ particle cluster. Beyond those edges, $S^{+}(r, N)$ has a linear gradient of order $1 / N$ and it is easy to show by a simple mean-field calculation that the average distance of the nearest downslope (upslope) bond from the right (left) edge of the particle cluster $\sim \sqrt{N}$. Therefore this downslope (upslope) bond can diffuse through this distance over a time-scale $\sim N$, and form a local hill, as shown in Fig. D-1A and then the downslope (upslope) bond moves ballistically down the valley, over a time-scale $\sim N$, and reaches the deepest point causing a unit displacement of the deepest point towards right (left). Thus the process illustrated in Fig. D-1 takes place over a time-scale $\sim N$ which explains the $D_{1} \sim 1 / N$ behavior for short times. The mechanism also shows that the particles do not undergo any displacement in this case. The deepest point of the valley slides back and forth beneath the $H$ particle cluster. This motion, however has an energy cost, and as discussed in the main text, for a distance $\Delta$ between the deepest point and the centre of mass of the $H$ cluster, the energy cost $\sim \Delta^{2}$. Thus the motion of the valley can be described by an Ornstein-Uhlenbeck process. This prevents the valley from taking a large excursion in either direction and as a result the mean-squared displacement reaches a plateau.

In the limit of very long time, the $H$ particle cluster will start moving around the system diffusively and the valley will naturally move along with it. This mechanism has been illustrated in Fig. D-2 which shows movement of an $L$ particle through the $H$ particle cluster. When the $L$ particle reaches the bottom of the valley, the deepest point undergoes a displacement, along with the center of mass of the $H$ particle cluster. Note that the time-scale for this 


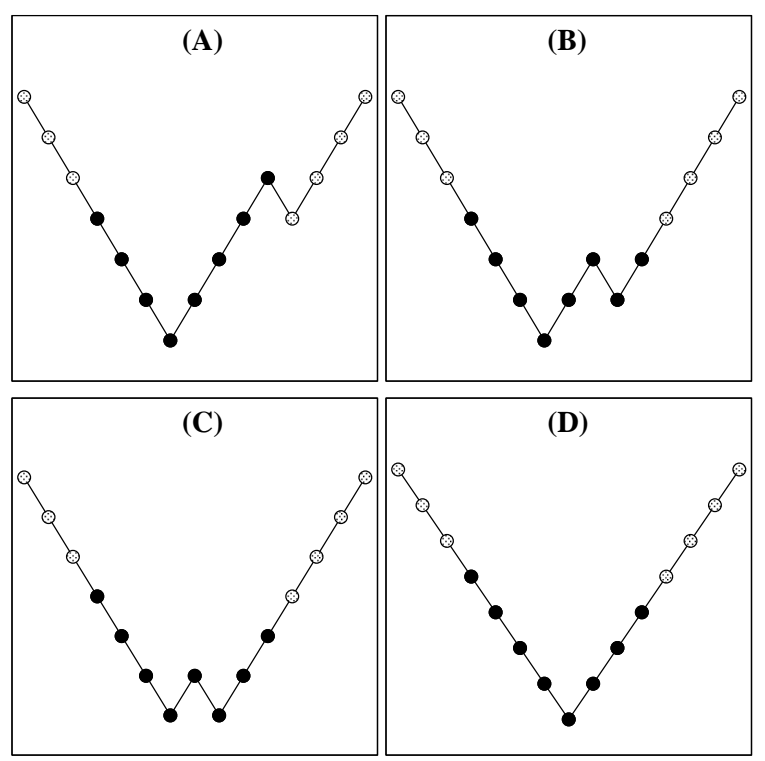

FIG. D-1: Motion of the deepest point over $\sim N^{2}$ time-scale. (A) An occupied local hill forms at the right edge of the particle cluster. (B) The occupied local hill, being unstable, zips through the pure domain of upslope bonds. (C) The local hill reaches the bottom of the valley. (D) The deepest point shifts one site to the right.

process is rather large because the probability that the $L$ particle, starting from the $H L$ domain boundary, reaches the bottom of the valley, is very low and decays exponentially with the domain size (see our data in Fig. D-3). As a result, the diffusivity of the valley in this regime $\sim e^{-\alpha N}$. Existence of an exponentially large time-scale breaks the translational invariance of the system but the relaxation time-scale is still algebraic, as demonstrated in our main text.

\section{Appendix E: Dynamical exponent for the FPS phase}

Two point density correlation function for the $H$ particles is defined as

$$
C(r, t)=<\rho_{i}(t) \rho_{i+r}(t)>-\rho_{0}^{2}
$$

where, $\rho_{i}(t)$ is $1(0)$ if the $i^{t h}$ site is occupied by an $H(L)$ particle at time $t$ during the coarsening phase. The angular brackets denote average over initial conditions. $\rho_{0}$ denotes the global density of $H$ particles in the system. Define $r_{0}$ as $C\left(r_{0}, t\right)=0$ and the dynamical exponent $z$ can be estimated from the variation of $r_{0}$ with $t$, using the scaling relation $r_{0} \sim t^{1 / z}$. The plots of $r_{0}$ vs $t$ for FPS phase in one and two dimensions are presented in Figs E-1 and E-2, respectively. 

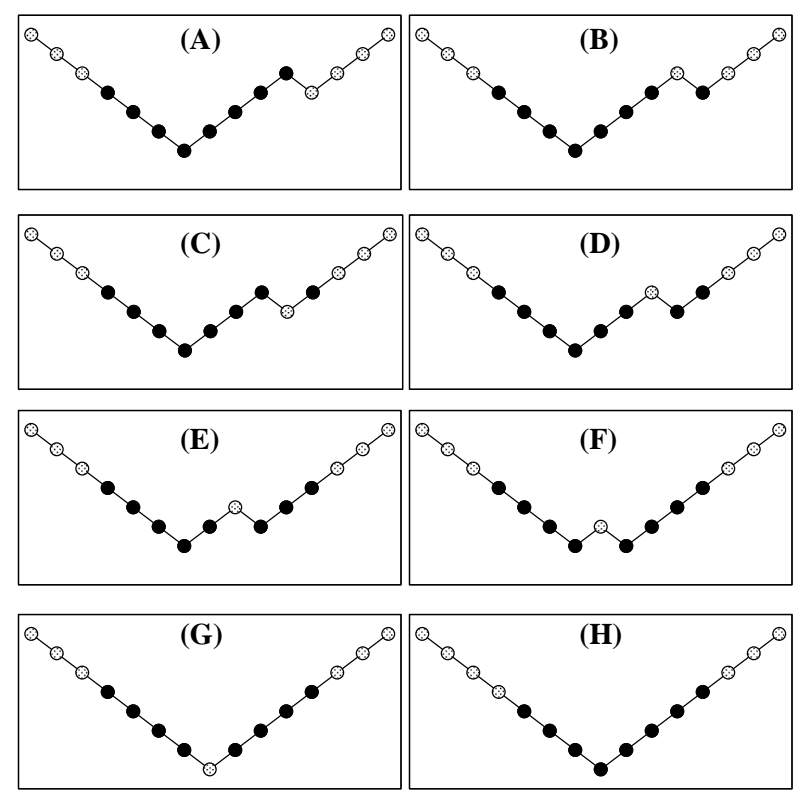

FIG. D-2: Motion of the deepest point over $\sim e^{N}$ time-scale. (A) Formation of a local hill at the right edge of the $H$ particle cluster. (B) The rightmost $H$ particle slides down the hill and gets detached from the cluster. (C) The resulting hill with $L$ particle flips and another local hill with $H$ particle is formed at the adjacent site. (D) Another $H$ particle slides down the hill and detaches from the $H$ cluster. (E) The $L$ particle propagates down the valley. (F) At the bottom of the valley a local hill with $L$ particle is formed. (G) This local hill flips and the deepest point of the valley shifts one site to the right. (H) The $H$ particles to the left of the deepest point slide down, one after another, moving the center of mass of the particle cluster to one site to the right.

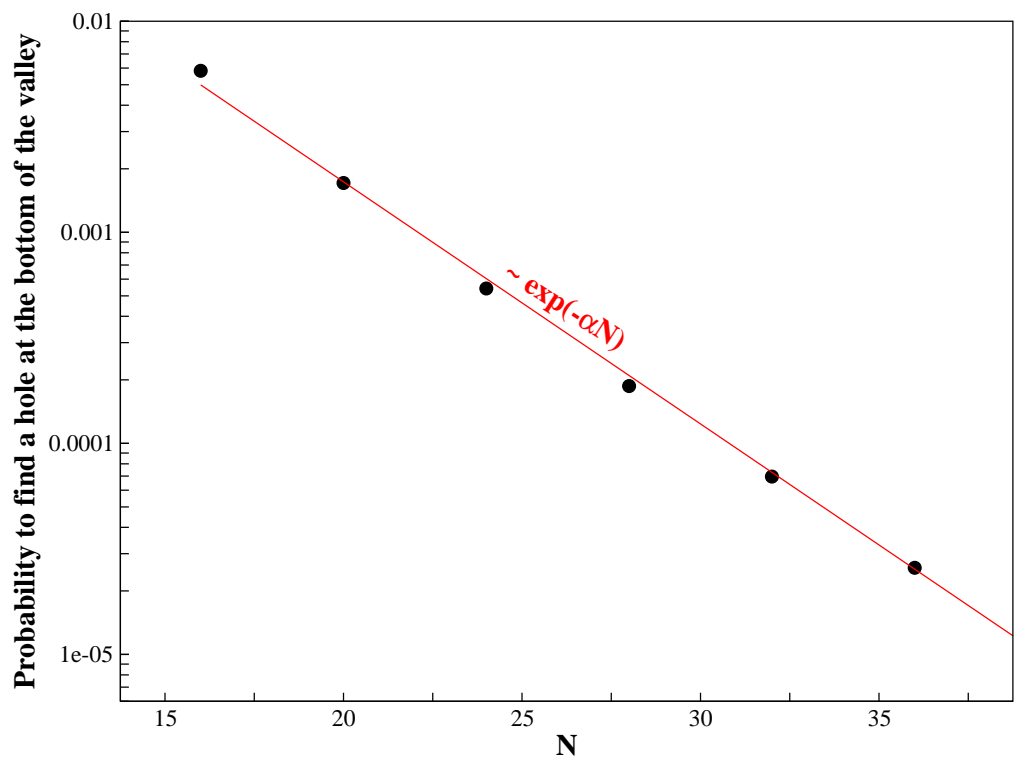

FIG. D-3: The probability to find an $L$ particle at the deepest point of the surface falls off as $e^{-\alpha N}$ with system size where $\alpha=0.261$. The data have been averaged over $10^{6}$ histories. 


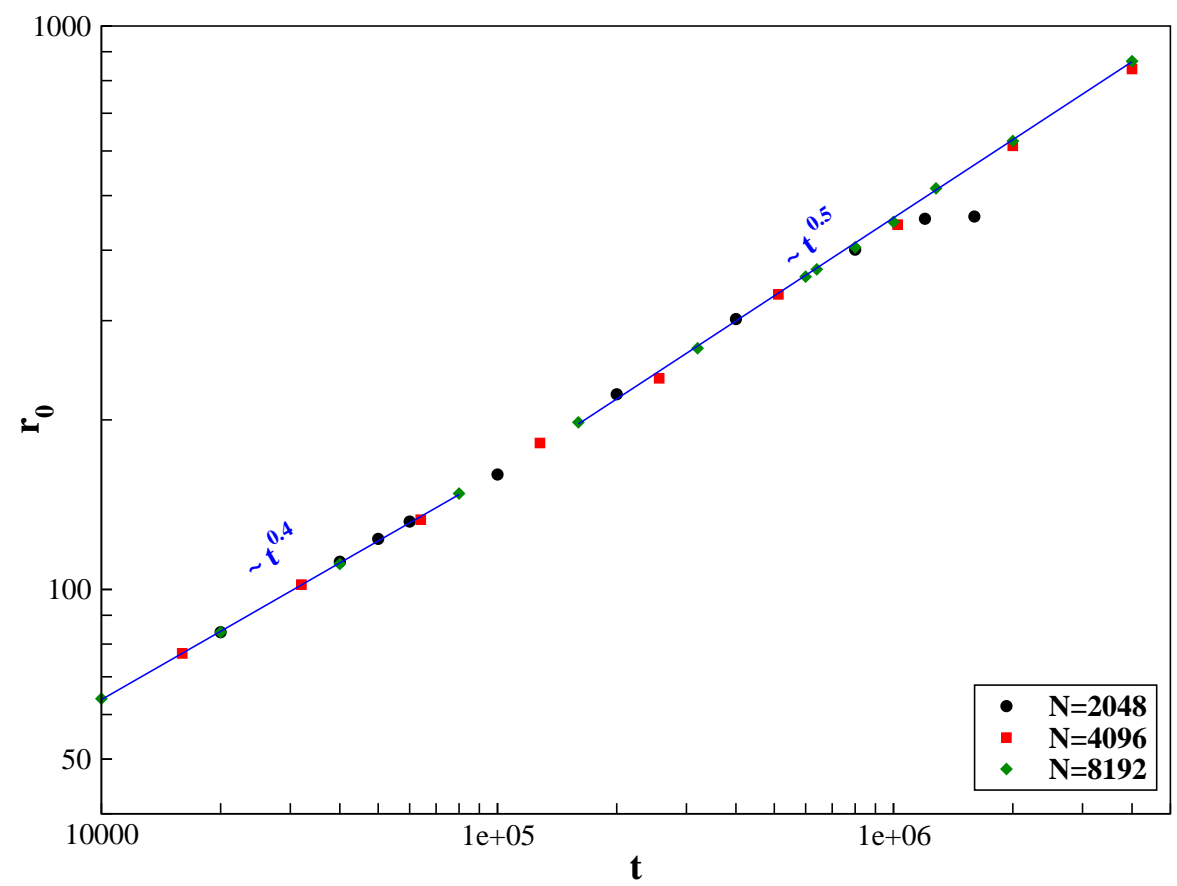

FIG. E-1: Plot of $r_{0}$ vs time during the coarsening phase for $b=0.3, b^{\prime}=-0.2$ in one dimension. For large $\mathrm{N}$ and $\mathrm{t}$, we obtain $r_{0} \sim t^{0.5}$. For $t \lesssim 10^{5}$, we observe a crossover region where $r_{0} \sim t^{0.4}$. We have used $\rho_{0}=1 / 2$ here.

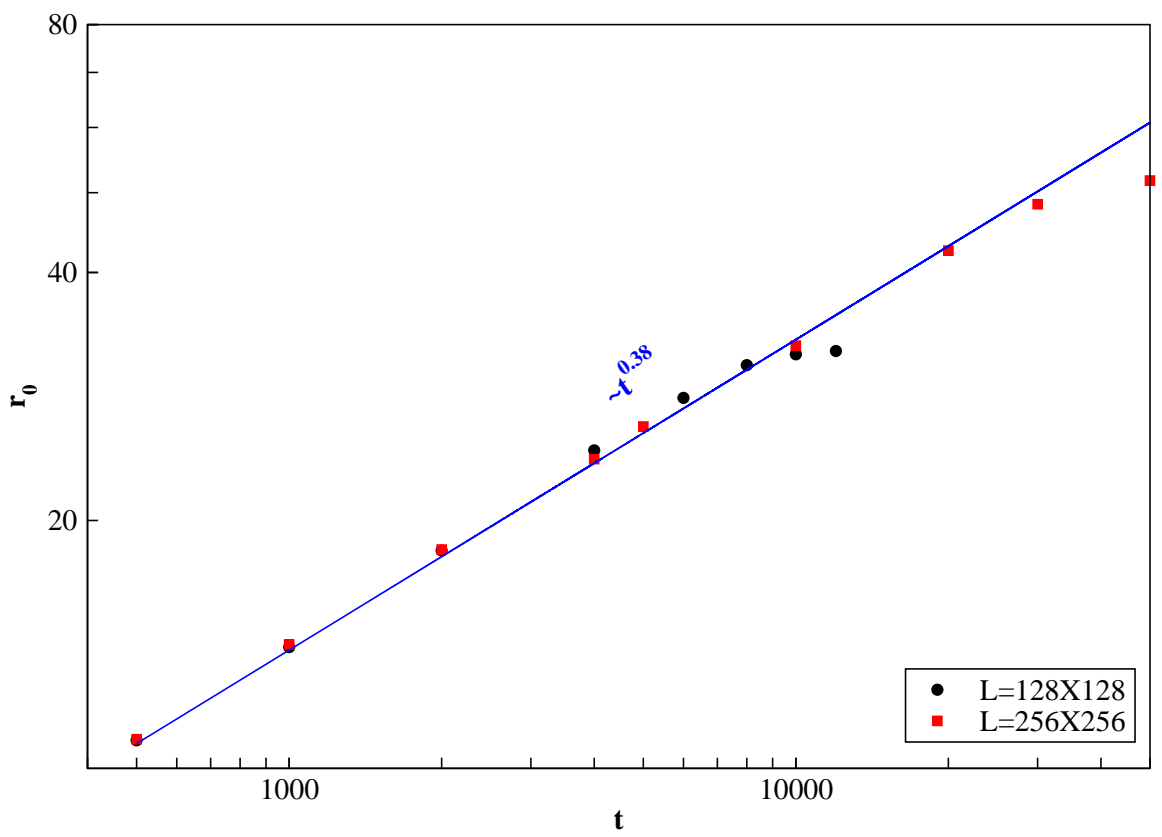

FIG. E-2: Plot of $r_{0}$ vs $\mathrm{t}$ during the coarsening phase for $b=0.3, b^{\prime}=-0.2$ in two dimensions. We obtain $r_{0} \sim t^{0.38}$. We have used $\rho_{0}=1 / 8$ here. 